\title{
The ATLAS Tile Calorimeter performance at LHC
}

\author{
Simon Molander \\ (On behalf of the ATLAS Collaboration) \\ Fysikum, Stockholm University, 10691 Stockholm, Sweden \\ simon.molander@fysik.su.se \\ Published 15 May 2014
}

\begin{abstract}
This paper gives an overview of the performance of the Tile Calorimeter of the ATLAS detector at the Large Hadron Collider. Detector performances with respect to electronic noise and cell response are presented. In addition, an overview of the partially overlapping calibration systems is given.
\end{abstract}

Keywords: Tile Calorimeter; calibration; performance; electronic noise.

\section{Introduction}

The Tile Calorimeter ${ }^{1}$ is an important sub-detector of the ATLAS experiment ${ }^{2}$ at the Large Hadron Collider. It is a scintillating calorimeter consisting of 5182 cells in total, layed out as shown in Fig. 1 for one of the 256 wedges of the detector. Each cell is read out using wavelength shifting fibers of both sides, giving redundancy. These fibers are connected to photo-multiplier tubes (PMT) which in turn are connected to the front end electronics where energy reconstruction is done.

\section{Calibration Systems}

The Tile Calorimeter is calibrated using multiple partially overlapping systems, ${ }^{2}$ giving stability and redundancy for the reconstructed signals. The gain variation for a cell as measured by some of these systems and compared with the integrated luminosity evolution are shown in Fig. 2.

The calibration of the electromagnetic scale was done using response to electrons in beam tests 2001-2004. ${ }^{3}$ The precision of the electromagnetic scale in any single cell is $3 \%$. This scale was then transferred into the ATLAS detector in the cavern and into deeper layers using the cesium system. ${ }^{4}$ The cesium system consists of three capsules containing ${ }^{137} \mathrm{Cs}$ sources that are pushed through the detector using a

This is an Open Access article published by World Scientific Publishing Company. It is distributed under the terms of the Creative Commons Attribution 3.0 (CC-BY) License. Further distribution of this work is permitted, provided the original work is properly cited. 


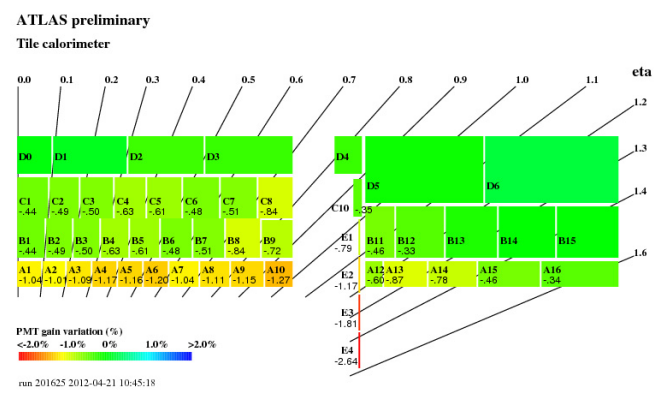

Fig. 1. Mean gain variation as measured by the laser system. Each numbered cell in the graph represents an average of 64 cells. This image also gives an overview of the detector layout, having four layers in the central barrel (to the left in the image), three in the end-cap (to the right) and six gap cells (left part of end-cap). The two intermediate layers in the barrel section (B and C) are read out as one.

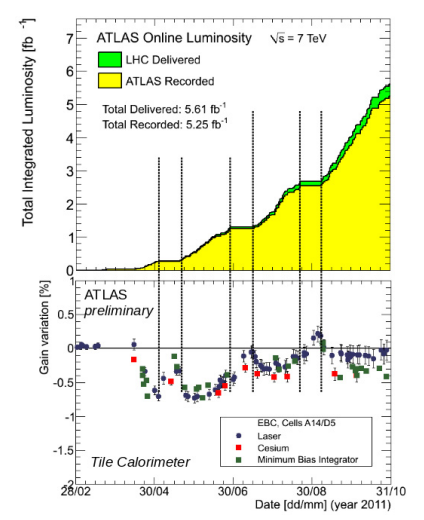

Fig. 2. Gain variation of a cell compared to total integrated luminosity. Measurements are done by three different systems.

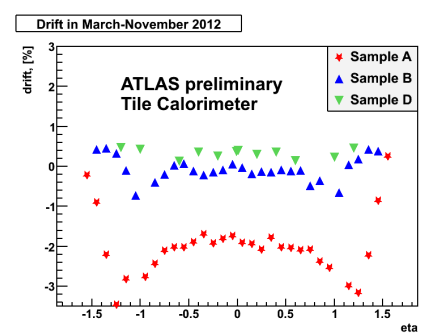

Fig. 3. Calibration drift in percent for for the cesium calibration system between March and November 2012 as a function of $\eta$, the different samples represent different radial layers as defined in Fig. 1 and every datapoint represents an average over 64 cells.

hydraulic system, thereby injecting signals into the scintillators. These calibrations are done on a monthly basis and can be used to calibrate and to monitor the full optics and photomultiplier response. The precision of the cesium calibration system is $0.3 \%$. The drift in calibration, mainly caused by variations in PMT gains, for the period between March and November 2012 is shown in Fig. 3 for each layer separately. In order to interpolate between cesium measurements a laser system ${ }^{2}$ is used on a weekly basis. The laser system works by injecting light pulses into the photo-multiplier tubes and thereby measures and monitors their gains. The precision of the laser calibration is better than $1 \%$. The PMT gain variation as measured by the laser system is shown in Fig. 1.

In order to convert the amplitude in $\mathrm{ADC}$ counts into a charge in $\mathrm{pC}$, a known charge is injected into the front end electronics. This is known as the charge injection 


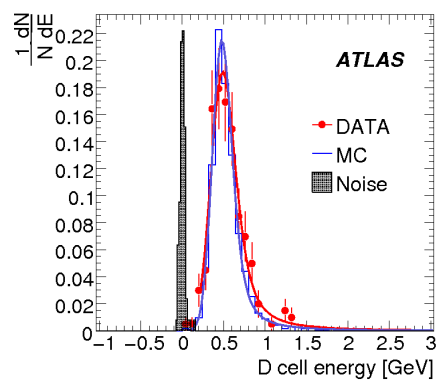

Fig. 4. Noise level compared to signal. The signal is from the response of Tile Calorimeter cells to cosmic muons.

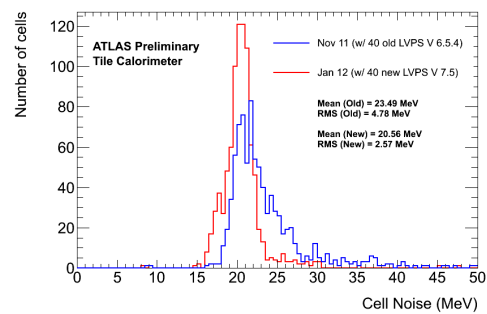

Fig. 5. New and more reliable low voltage power supplies were installed during a maintenance period. They improved the cells' noise as shown by the red line (new) as compared to the blue line (old). Cells with a new power supply have a lower mean noise and a Gaussian distribution.

system (CIS). ${ }^{2}$ This is done on a weekly basis, and the constants have been shown to be highly constant and are therefore only updated twice a year. The precision of the CIS calibration is $0.7 \%$.

\section{Electronic noise}

The signal-to-noise ratio is a good indicator of detector performance. In addition, knowledge of noise rates is important for reconstruction of constituents of higher level objects such as topological clusters. Figure 4 shows a comparison of signal and noise done by studying cosmic muons. ${ }^{5}$ Noise levels were reduced when the low voltage power supplies (LVPS) where replaced during a maintenance period. ${ }^{6}$ The result of this replacement can be seen in Fig. 5 .

\section{Calorimeter Response}

The calorimeter energy response can be evaluated by measuring how much energy deposited by a single hadron of a given momentum measured in the inner tracker, expressed as $\frac{E}{p}$ where $\mathrm{p}$ is a track momentum. For this evaluation, isolated tracks having energy deposits compatible with minimum ionizing particles in the electromagnetic calorimeter, which is in front of the Tile Calorimeter, are selected. The data is also compared to Monte Carlo simulations. Results are shown as a function of momentum in Fig. 6 and as a function of the angle $\phi$ in Fig. 7. The largest disagreement between data and Monte Carlo is $12 \%$, for $11<p<12 \mathrm{GeV}$.

\section{Conclusions}

Performance evaluations based on calibration systems, cosmic muons and collision data have been presented. The Tile Calorimeter has redundancy in both read-out and calibration systems. The cell response is monitored and adjusted to the level 


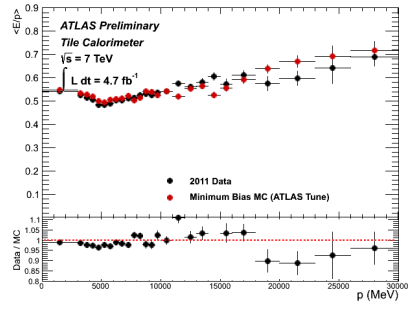

Fig. 6. Calorimeter Response characterised by energy over momentum (E/p) for isolated tracks using proton-proton collision data from 2011 in the Minimum Bias stream. The plot shows the mean of the $\mathrm{E} / \mathrm{p}$ ratio for hadronic tracks as a function of phi, integrated over the $|\eta|<0.7$.

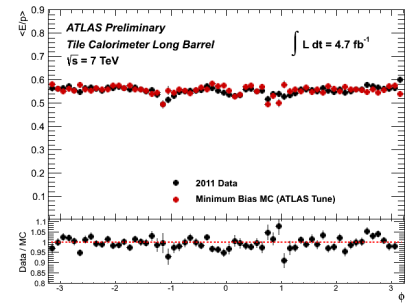

Fig. 7. Calorimeter Response characterised by energy over momentum (E/p) for isolated tracks using proton-proton collision data from 2011. The plot shows the mean of the $\mathrm{E} / \mathrm{p}$ ratio for hadronic tracks as a function of momentum, integrated over $\phi$. The lower plot shows the ratio of data to simulation in the same momentum bins.

of $\sim 1 \%$ and in situ energy response validation shows agreement with the hadronic shower simulation prediction within a few percent.

\section{Acknowledgments}

In addition to the ATLAS collaboration as a whole, I would like to express my acknowledgements to: My thesis advisor, Sten Hellman, who helped out a lot with technical and practical considerations. Olle Lundberg and Pawel Klimek who helped me with templates and formatting of the poster itself. Eirini Vichou and Tomas Davidek for helping me with improvements.

\section{References}

1. G. Aad et al., The ATLAS Experiment at the CERN Large Hadron Collider, J. Instrum., 3:S08003. 437 p., 2008. Also published by CERN Geneva in 2010.

2. E. Berger, R. Blair and J. Dawson, Construction and performance of an iron-scintillator hadron calorimeter with longitudinal tile configuration, Technical Report CERNLHCC-95-44. LRDB-RD-34, CERN, Geneva, 1995.

3. P, Adragna et al. Testbeam Studies of Production Modules of the ATLAS Tile Calorimeter, Nucl. Instrum. Methods Phys. Res., A, 606 (ATL-TILECAL-PUB-2009002. ATL-COM-TILECAL-2009-004. 3):362-394. 73 p., Feb 2009. revised version, includes new author lists.

4. E. A. Starchenko, G. Blanchot et al., Cesium Monitoring System for ATLAS Tile Hadron Calorimeter, Technical Report ATL-TILECAL-2002-003, CERN, Geneva, Feb 2002.

5. G. Aad et al., Readiness of the ATLAS Tile Calorimeter for LHC collisions, Eur. Phys. J., C70:1193-1236, 2010.

6. G. Drake, T. Cundiff et al., An Upgraded Front-End Switching Power Supply Design for the ATLAS TileCAL Detector of the LHC, Technical Report ATL-TILECAL-PROC2011-015, CERN, Geneva, Nov 2011. 